\title{
Numerical Simulation of Flow and Determination of Aerodynamic Forces in the Balanced Control Valve
}

\author{
R. Matas ${ }^{1, \mathrm{a}}$, F. Straka ${ }^{1}$ and M. Hoznedl ${ }^{2}$ \\ ${ }^{1}$ University of West Bohemia in Pilsen, Univerzitni 8, 30614 Plzen, Czech Republic \\ ${ }^{2}$ SKODA POWER s.r.o., Tylova 1/57, 30128 Plzen, Czech Republic
}

\begin{abstract}
The contribution subscribes a numerical simulation of a steam flow through a balanced control valve. The influence of some parameters in simulations were tested, analyzed and discussed. As a result of the simulations a graph of aerodynamics forces for a specific turbine characteristic was obtained. The results from numerical simulations were compared with results from experiments. The experiment was performed with an air flow, but the final data were converted with a criterion to steam flow.
\end{abstract}

\section{Introduction}

Development of a new type of valve for steam turbines raises a number of questions regarding its flow and force characteristics. In order to determine the behaviour of the newly designed valve intended even for large outputs and high steam parameters design methods, CFD simulation and experimental measuring on models are applied [1,3]. The objective is to secure low losses, reliability within a wide range of operation modes and a suitable force characteristic for controlling.

\section{Control valves for steam turbines}

A control valve $(\mathrm{CV})$ of a steam turbine regulates the flow rate of steam entering the turbine and thus affects its output. This valve is adjustable within the whole control range and offers a full range of opening and in this way the required flow rate of steam to the turbo set is achieved.

\subsection{Basic variables}

The flow through a control valve can be comprehended as a flow through a nozzle. The patterns and principles occurring here are the same, but the geometry of a nozzle is much more simple than the geometry of a control valve and therefore the actual behaviour of the control valve may be stated by means of relations of a nozzle only approximately as 3D effects are applied here.

a e-mail: mata@ntc.zcu.cz
The theoretical critical mass flow, which is determined by the formula:

$$
\dot{m}_{k r}=\left[2 \cdot\left(i_{1 C}-i_{k r}\right)\right]^{\frac{1}{2}} \cdot \rho_{k r} \cdot \frac{\pi \cdot D_{h}^{2}}{4},
$$

is an important value; for ideal gas, it can be replaced with the formula:

$$
\dot{m}_{k r}=S^{*} \cdot \sqrt{\frac{p_{0}}{v_{0}}} \cdot\left(\frac{2}{\kappa+1}\right)^{\frac{1}{\kappa-1}} \cdot \sqrt{\frac{2 \kappa}{\kappa+1}} .
$$

The proportional mass flow is determined by the formula (3); it relates the instantaneous mass flow to the critical mass flow.

$$
q=\frac{\dot{m}}{\dot{m}_{k r}} .
$$

The pressure ratio is a ratio between the static pressure on the outlet from the control valve and the total pressure on the valve inlet. It is determined by the formula:

$$
\varepsilon=\frac{p_{V}}{p_{0}} .
$$

The proportional travel height is a real travel of the valve plug related to the critical diameter of the nozzle. It is determined by the equation:

$$
\bar{h}=\frac{H}{D} .
$$

\subsection{Balanced and unbalanced valves}

In terms of the requirement of the highest efficiency and reliability of these elements there are a number of designs of control valves corresponding to the use under the particular specified conditions. 
Control valves may differ based on several criteria, e.g. by the shape of the plug, number of seating faces, etc. We will be interested in the division according to the arrangement of force that needs to be applied to the plug in order to change the position of the plug from closed to open. According to this, we divide control valves into balanced and unbalanced. If the force equals the force corresponding with the product of the annular area and the difference of pressures before and after the valve, the valve is unbalanced; if the force is lower, it is a balanced valve. The paper [2] describes results of calculations and measurements of flow-through characteristics of unbalanced valves with varying seat inflow angle and concepts corresponding to the further stated valves. In the following stage, research focused on phenomena in the balancing system and in particular the force characteristics of the plug of this balanced single-seat diffuser control valve.

\subsection{Balanced valve design}

To actuate a CV it is necessary to apply the force required for changing of valve position. Servomotors that actuate the $\mathrm{CV}$ must be designed for these forces. In the case of high input pressure values, these forces may be substantial and therefore there is a tendency to reduce these forces. This may be done in several ways, e.g. an internal bypass valve, external bypass valve or a valve with a permanent leak.

The force affecting the plug from pressures before $(p 0)$ and after the valve $(p V)$, where $\mathrm{d}$ is the diameter of the surface on which the said pressures act:

$$
F=\pi \cdot d^{2} \cdot(p O-p V) / 4
$$

In the case of the examined valve, it is a $\mathrm{CV}$ with internal bypass where the pressure is reduced. Therefore the resulting force to the plug does not reach such values as it is in the case of unbalanced CVs. The design of the modelled valve or its key inner part is shown in figure 1 .

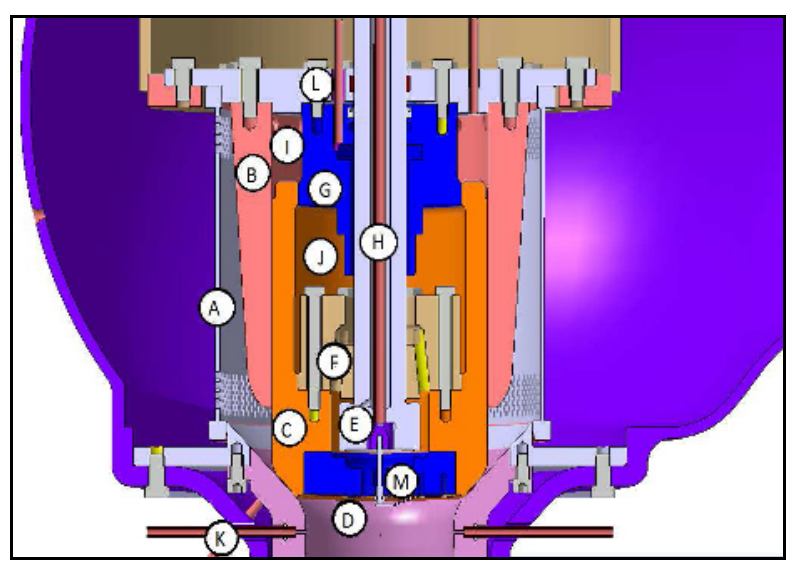

Fig. 1. Detail of balanced CV section

Part A shows a screen which directs and equalizes steam flow in particular in the case of high travel. B is a sleeve with holes allowing steam passage into the internal part of the valve. I marks the 1 st chamber and $\mathrm{J}$ the 2 nd chamber. A $0.15 \mathrm{~mm}$ slot is placed between these chambers. Using part $\mathrm{G}$, the position of closing of $\mathrm{J}$ and $\mathrm{I}$ chambers is set by default. $\mathrm{C}$ is the main plug sitting on the seating face of the diffuser. If travel exceeds $13 \mathrm{~mm}$, a 2nd slot is created between the $\mathrm{J}$ and $\mathrm{F}$ parts, which has the same clearance as the 1 st slot, i.e. $0.15 \mathrm{~mm}$. Part F separates the 2 nd chamber from the bypass part of the plug E. This bypass plug is directly connected with the shaft $\mathrm{H}$ controlled by the servo-drive. Steam is let out through part $\mathrm{M}$, which contains several holes, by balancing under the plug and further into the diffuser.

Areas K, L and M designate the areas of measuring using pressure bleeding and strain gauges during experimental examination of the model valve; they are not present at valves in practical applications.

\section{Computational model}

Numerical simulation was performed on the described balanced valve using the ANSYS/FLUENT system; only a few slight geometric modifications were performed on the model as more significant modifications would further complicate the already difficult simulation.

\subsection{Geometry}

Valve geometry was adjusted on the CAD model and completed in the ANSYS 13.0 system by the module Design Modeler.

The created geometry contains parameters allowing regulation of valve travel. This is advantageous for numerical analysis of any required travel without further complex adjustment of geometry. The created model allows controlling by means of parameters both the travel of the large plug within the scope $0-34 \mathrm{~mm}$ and the travel of the small bypass plug within the full travel range $0-6 \mathrm{~mm}$. In the case of some travel lengths when surfaces overlap, slight manual interventions are required. Geometry was further adjusted so that the very complex inner bypass part of the valve and its subsequent coverage by the computing mesh were also taken into account; the result is available in figure 2 .

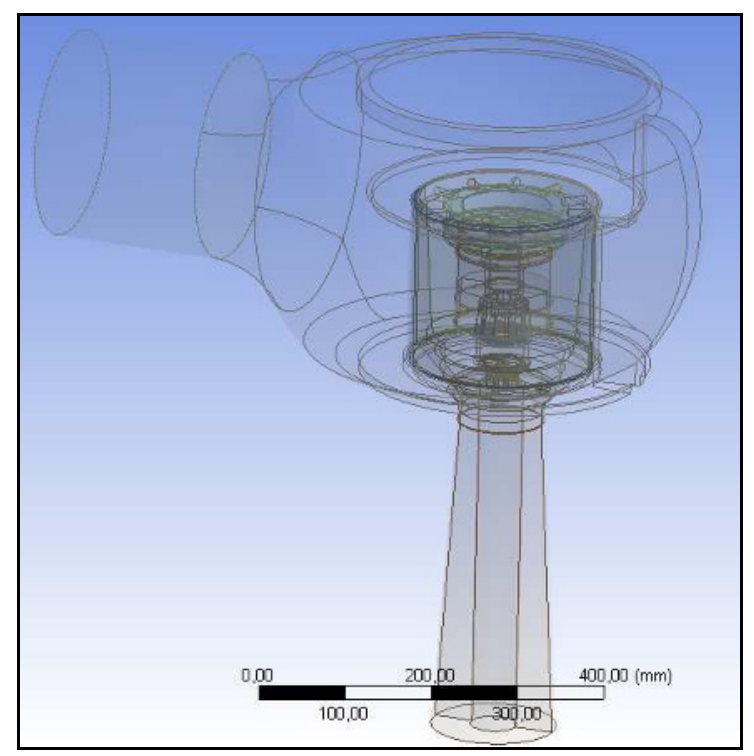

Fig. 2. CFD Geometry of valve model 


\subsection{Computational mesh}

The mesh was created in Meshing module. When creating the mesh, it was revealed that the inner algorithms of the meshing are not quite suitable for more complex and significantly articulated geometry. When solving a similar task, it would be much more suitable to use another mesh generator allowing to suitably cover in particular the area of slots and transitions to them (ICEM/CFD, GAMBIT, etc.).

Despite that, suitable computational meshes were eventually produced for all required travels. These were 12 different travel lengths [mm]: $0,2,6,10,11.5,13,15$, $16,18,20,27.4$ and 30 . These were hybrid computational meshes with the majority of six-plane cells and the total count of ca 3.5 million per each travel. At places where significant speed or pressure gradients were expected, the mesh was adjusted accordingly. A sample of a mesh for one solved travel is provided in figure 3 .

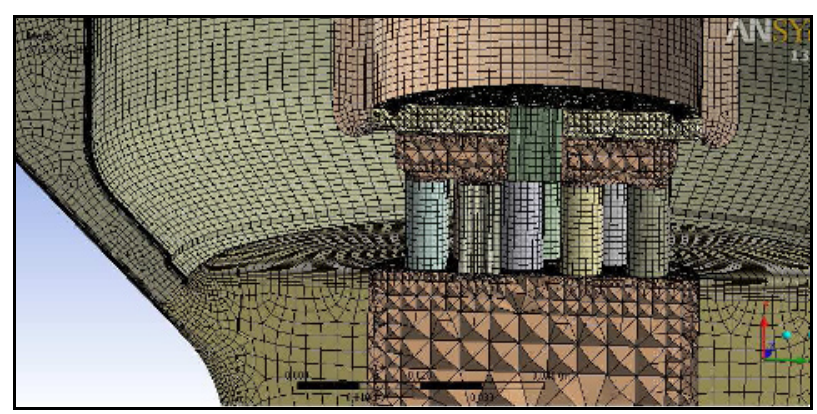

Fig. 3. Computational mesh for CFD simulation

\subsection{Medium parameters}

The medium in computation was steam with properties of an ideal gas. The properties of the medium are different for individual travel lengths and were entered according to the used computation methodology in order to suitably substitute for actual steam behaviour; table 1 shows the used values of physical properties and limit conditions.

Table 1. Steam parameters at individual travel lengths

\begin{tabular}{|c|c|c|c|c|c|c|}
\hline $\begin{array}{l}\text { Plug travel } \\
\text { (mm) }\end{array}$ & 0 & 2 & 6 & 10 & 11.5 & 13 \\
\hline po (MPa): & 1.4291 & 1.4291 & 1.4291 & 1.4291 & 1.4291 & 1.4291 \\
\hline $\mathrm{pV}$ (MPa): & 0.0214365 & 0.242947 & 0.8031542 & 1.0989779 & 1.1704329 & 1.2218805 \\
\hline$\varepsilon$ & 0.0150 & 0.1700 & 0.5620 & 0.7690 & 0.8190 & 0.8550 \\
\hline$t\left({ }^{\circ} \mathrm{C}\right):$ & 326.24 & 326.24 & 326.24 & 326.24 & 326.24 & 326.24 \\
\hline $\mathrm{M}_{\mathrm{m}}(\mathrm{kg} / \mathrm{kmol}):$ & 18.551 & 18.551 & 18.556 & 18.562 & 18.564 & 18.565 \\
\hline $\mathrm{R}(\mathrm{J} / \mathrm{kg} / \mathrm{K}):$ & 448.2 & 448.2 & 448.0 & 447.9 & 447.9 & 447.8 \\
\hline$c_{v}(J / k g / K):$ & 1929.5 & 1929.5 & 1932.5 & 1935.8 & 1937.1 & 1938.1 \\
\hline $\mathrm{v}(\mathrm{Ns} / \mathrm{m} 2)$ & $1.9628 \mathrm{E}-05$ & $1.9628 \mathrm{E}-05$ & $2.0128 \mathrm{E}-05$ & $2.0527 \mathrm{E}-05$ & $2.0700 \mathrm{E}-05$ & $2.0822 \mathrm{E}-05$ \\
\hline$\lambda(W / m / K)$ & 0.043387 & 0.043387 & 0.044387 & 0.045712 & 0.046134 & 0.046501 \\
\hline $\begin{array}{l}\text { Plug travel } \\
\text { (mm) }\end{array}$ & 15 & 16 & 18 & 20 & 27.4 & 30 \\
\hline po (MPa): & 1.4291 & 1.4291 & 1.4291 & 1.4291 & 1.4291 & 1.4291 \\
\hline $\mathrm{pV}$ (MPa): & 1.271899 & 1.2876191 & 1.3176302 & 1.3347794 & 1.3562159 & 1.3636 \\
\hline$\varepsilon:$ & 0.8900 & 0.9010 & 0.9220 & 0.9340 & 0.9490 & 0.9540 \\
\hline $\mathrm{t}\left({ }^{\circ} \mathrm{C}\right):$ & 326.24 & 326.24 & 326.24 & 326.24 & 326.24 & 326.24 \\
\hline$M_{m}(\mathrm{~kg} / \mathrm{kmol}):$ & 18.566 & 18.567 & 18.567 & 18.568 & 18.568 & 18.569 \\
\hline $\mathrm{R}(\mathrm{J} / \mathrm{kg} / \mathrm{K}):$ & 447.8 & 447.8 & 447.8 & 447.8 & 447.7 & 447.7 \\
\hline$c_{0}(\mathrm{~J} / \mathrm{kg} / \mathrm{K}):$ & 1939.0 & 1939.3 & 1939.8 & 1940.1 & 1940.8 & 1940.9 \\
\hline $\mathrm{v}(\mathrm{Ns} / \mathrm{m} 2)$ & $2.0970 \mathrm{E}-05$ & $2.0970 \mathrm{E}-05$ & $2.1036 \mathrm{E}-05$ & $2.1068 \mathrm{E}-05$ & $2.1120 \mathrm{E}-05$ & $2.1150 \mathrm{E}-05$ \\
\hline$\lambda(W / m / K):$ & 0.046802 & 0.046901 & 0.047080 & 0.047100 & 0.047350 & 0.047420 \\
\hline
\end{tabular}

The entered values are as follows: steam pressure at outlet from diffuser $p V$, pressure ratio $\varepsilon$, molecular mass
$M_{m}$, gas constant $R$, specific heat $c_{p}$, dynamic viscosity $v$, heat conductivity $\lambda$.

The universal parameters are as follows: steam inlet pressure $p O=1.429 \mathrm{MPa}$ and inlet steam temperature $t=326^{\circ} \mathrm{C}$.

\subsection{Numerical simulation}

Flow simulation was performed using the ANSYS/FLUENT v. 13 commercial CFD code. The considered flow was single-phase, compressible and turbulent for all solved cases. Based on the results of previous studies [1] the RNG k- $\varepsilon$ turbulence model was used and the combined implicit solver with second order schemes. It was necessary to use the Full Multigrid (FMG) type initialization and the Courant Number value had to be lowered to 2. Calculations were performed on the computational cluster; when using 8 cores (Xeon $\mathrm{X} 5680$ ) the computation time of one variant was $40-60$ hours.

\subsection{Calculated flow patterns}

The process of computations was stable, the resulting flow in the actual valve was basically stationary with a few exceptions. Non-stationary phenomena were observed with $2 \mathrm{~mm}, 27.4 \mathrm{~mm}$ and $30 \mathrm{~mm}$ travel lengths.

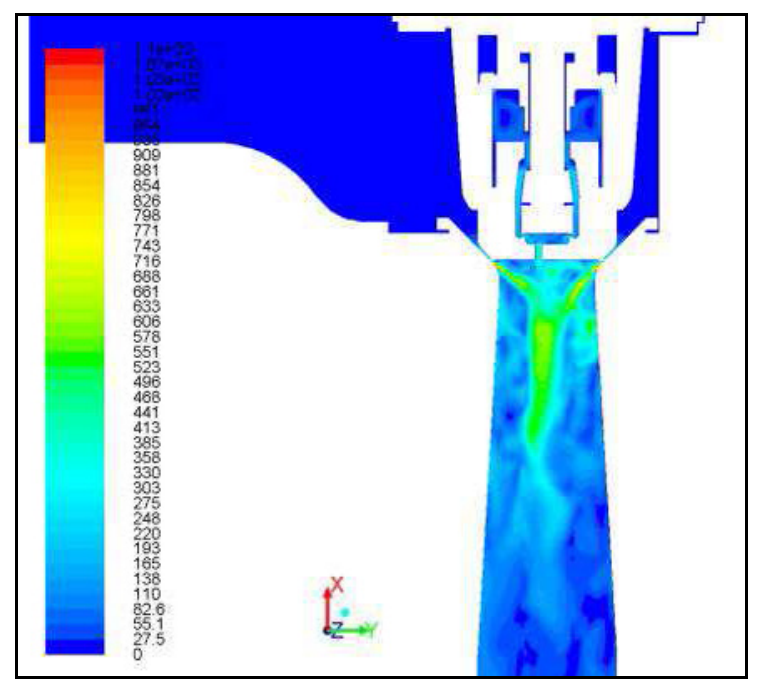

Fig. 4. Travel $2 \mathrm{~mm}$, velocity distribution $\left[\mathrm{m} . \mathrm{s}^{\mathrm{I}}{ }^{1}\right]$

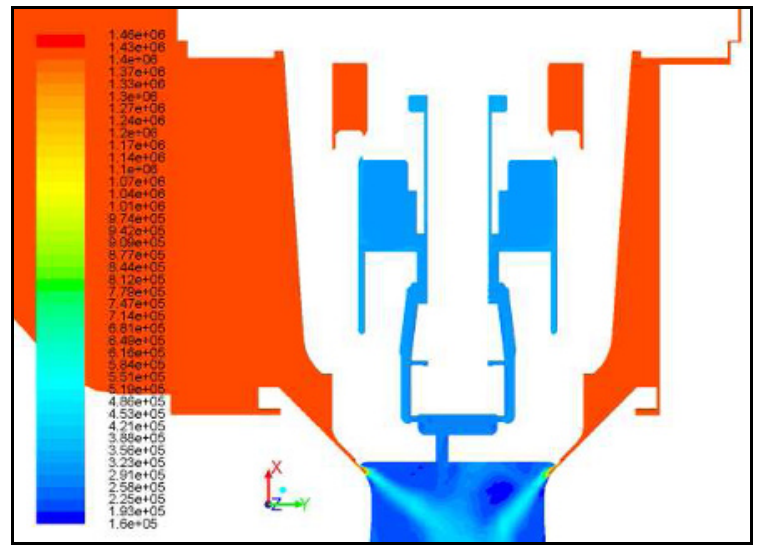

Fig. 5. Travel $2 \mathrm{~mm}$, overall pressure distribution [Pa] 
In the case of $2 \mathrm{~mm}$ (figure 4 and 5) travel, the great pressure change under the plug causes pulsation of flow and separation of steam flow from the diffuser wall.

In the case of $6 \mathrm{~mm}$ travel, figures 6 and 7 show flow stabilization in the valve and adhering of the flow to the diffuser wall. This flow condition is desirable for the valve as it stabilizes the flow and eliminates valve vibrations, as was proved by previously performed research of valve behaviour [1].

The $11.5 \mathrm{~mm}$ travel length also preserves a similarly stable flow nature as may be seen in figures 8 and 9. In terms of flow patterns, the picture does not differ much for other travel lengths up to $27.4 \mathrm{~mm}$.

In the case of 0,2,6 and $11.5 \mathrm{~mm}$ travel lengths, local supersonic velocity occurs formed due to flow through a nozzle in the case of an off-design mode.

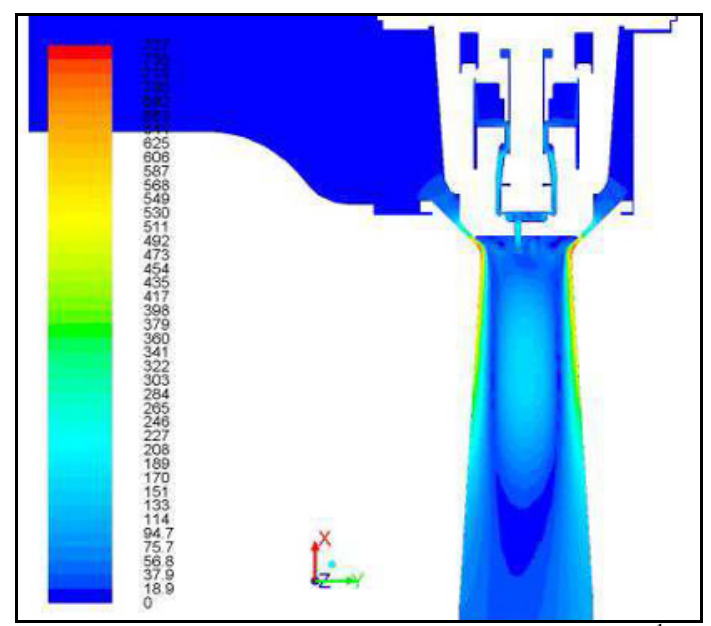

Fig. 6. Travel $6 \mathrm{~mm}$, velocity distribution $\left[{\mathrm{m} . \mathrm{s}^{-}}^{1}\right]$

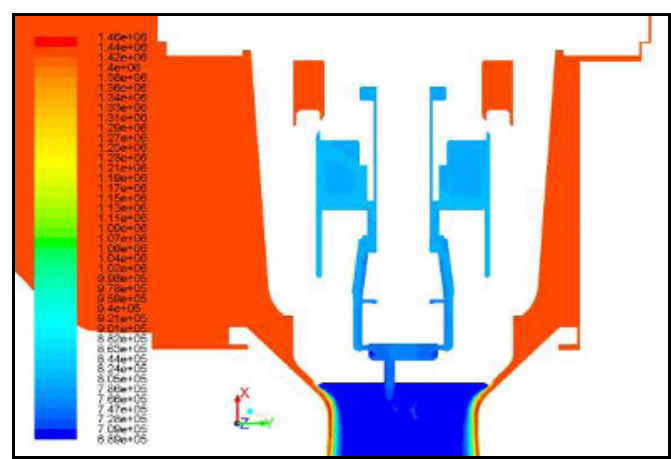

Fig. 7. Travel $6 \mathrm{~mm}$, overall pressure distribution $[\mathrm{Pa}]$

In the case of long travel lengths $(27.4$ and $30 \mathrm{~mm})$ with a high flow rate, less stable flow occurs which is well illustrated by figures 10 and 11 . The non-stationary character is not a problem here in terms of vibrations, as in the case of travel lengths near the full opening, low pressure gradients are present in the valve. In terms of losses the situation is slightly worse and it is the price for using the plug with a flat bottom, which is suitable in terms of dynamics in off-design conditions with the low opening and pressure ratio.

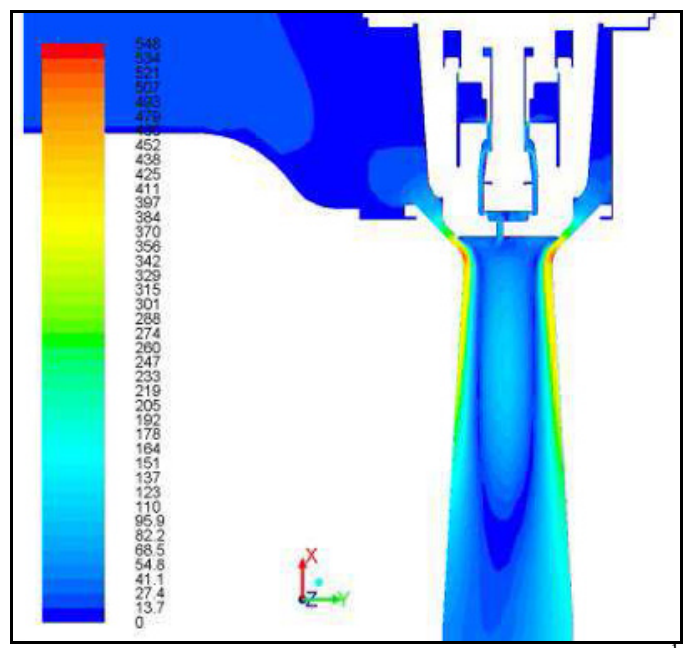

Fig. 8. Travel $11.5 \mathrm{~mm}$, velocity distribution $\left[{\mathrm{m} . \mathrm{s}^{-}}^{\mathrm{I}}\right]$

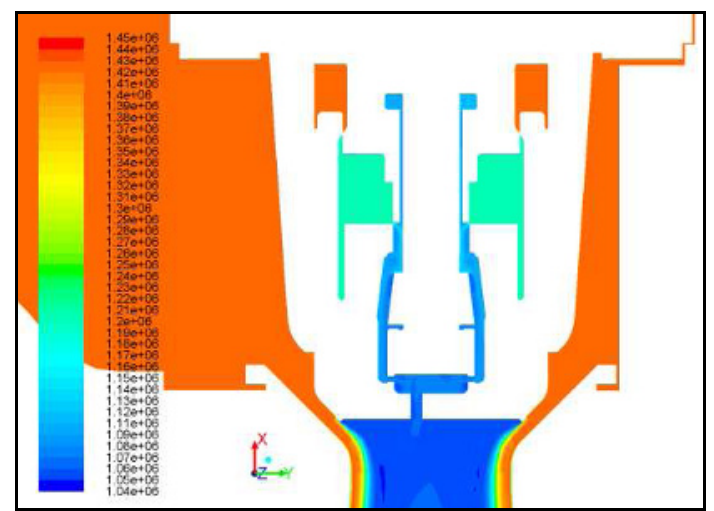

Fig. 9. Travel $11.5 \mathrm{~mm}$, overall pressure [Pa]

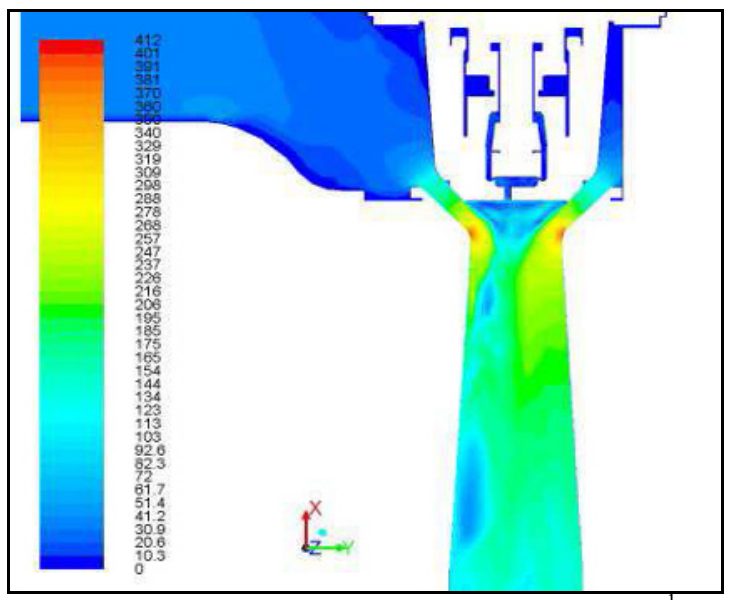

Fig. 10. Travel $30 \mathrm{~mm}$, velocity distribution $\left[{\mathrm{m} . \mathrm{s}^{-1}}^{1}\right]$

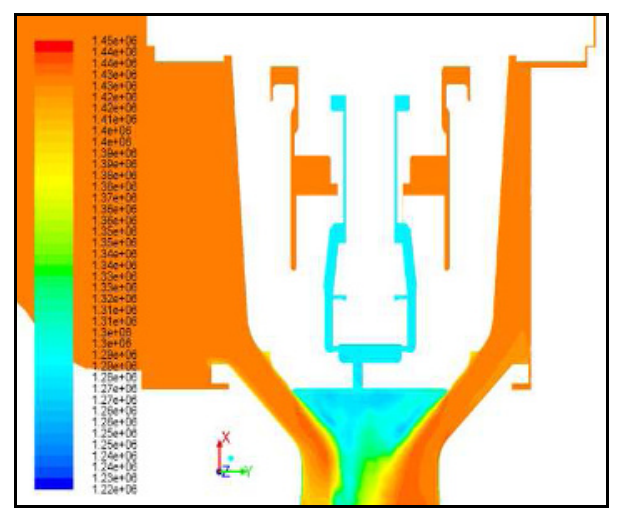

Fig. 11. Travel $30 \mathrm{~mm}$, overall pressure distribution $[\mathrm{Pa}]$ 
The results show that flowing through narrow leak using FLUENT software may be solved; however the question for other studies is how great the deviation from the actual conditions is and it is possible to compare pressures in the system by experimentation.

The flow rate through balancing is negligible compared to the flow rate through the whole valve and it is in single percentages.

\section{Experimental measuring}

The model valve is located in an air tunnel of the ŠKODA Power s.r.o. experimental laboratory. The tunnel has an open cycle and the air is supplied by a radial compressor with regulation.

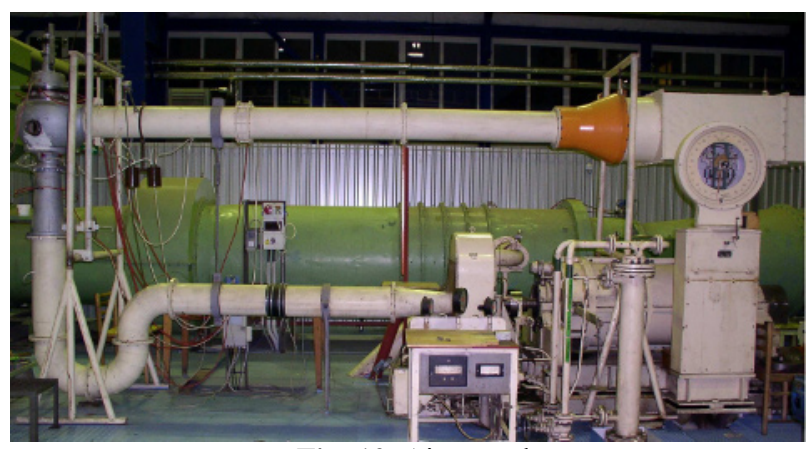

Fig. 12. Air tunnel

The equipment shown in figure 12 is used for measuring flow characteristics; its design allows measurement of force characteristics, i.e. load on the plug or the shaft.

Using sampling it is possible to measure all required pressure values at various places of the valve. The bypass plug is connected with the large plug by a draw rod, on which strain gauges are placed and it is used as a measuring element. The bypass valve is further connected with the lifting mechanism using another measuring element on which strain gauges are also attached. The reason for measuring by these strain gauges is to determine the stabilization force acting on the plug. This force must be the greatest when opening the valve, in order to secure stability of the CV. The second strain gauge is used for determination of the force on the servodrive lifting the plug.

The flowing medium in this case is air; conversion to actual parameters is performed using dimensionless characteristic numbers.

\section{Force characteristic}

The intersections of flow characteristic of the valve with the flow characteristic of the turbine are the basis for design of points in which forces are examined and from which force characteristic is determined. The flow characteristics of the valve were measured on an experimental model valve, the flow characteristic of the turbine was determined for the assumed turbine the parameters of which were used for design calculation of the valve. These characteristics are shown in figure 13 and are the basis for parameters for CFD numeric simulation and for measuring forces on the experimental model.

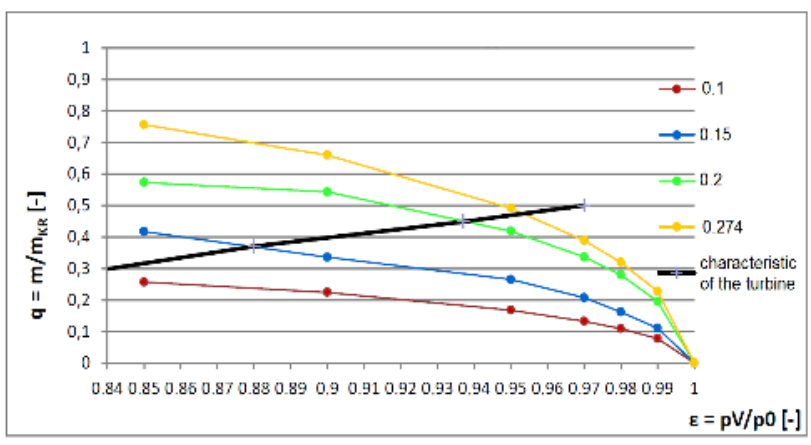

Fig. 13. Flow characteristic of the valve and considered characteristic of the turbine

The force characteristic was created from simulations using deduction of pressure values in those parts of the valve which act on the main or bypass part of the valve. The result is the curve marked CFD - calculation in the diagram in figure 14. The other two curves mark the results of experimental measuring. The results of measuring differ according to the method of ascertainment of forces. The first method was direct determination of the force using a strain gauge fixed on a certain part of valve. Another way of measuring was determination of pressure values using pressure bleeds and subsequent conversion via surfaces on which the pressure values act.

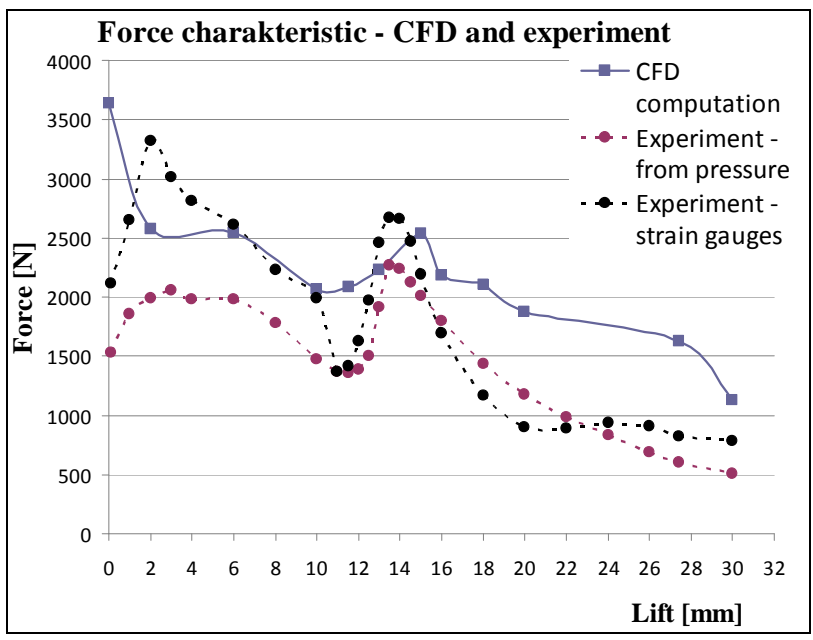

Fig. 14. Force characteristic

When looking directly at the course of forces in the diagram it may be said that the characteristic of the force exactly copies the curves obtained in an experimental way. However, the value of the curve at point 0 does not correspond to the value from the experiment. This is given by the method of experimental measuring. It was impossible to measure pressure values as low as this and the values were obtained by interpolation. However, as may be seen, this method markedly differs from the result from numerical simulation.

Further, it is possible to infer from the diagram that the largest force acts on the plug during zero travel. At 
this travel the bypass plug is open. The force diagram has a globally descending tendency, which indicates that with increasing travel height, the force on the shaft due to aerodynamic forces decreases. There is an interesting area in the diagram between travel values 12 and 18 , when the stabilization force locally increases. This is given by the balancing function which ceases to act at this stage. It is the correctly designed geometry in this area which is key for the force characteristic.

Further, it is worth noticing the point 2, which is markedly under the expected value. It may be given by the fact that (as was shown by flow pattern values from simulations) flow is non-stationary here and the flow is detached from the diffuser. The question is whether these circumstances may affect the solution to this degree; the used computation mesh and its unsuitable configuration in the areas of balancing system slots may also be blamed.

\section{Conclusion}

Using numerical simulation performed by the ANSYS/FLUENT system, flow patterns were obtained from steam flow in a balanced control valve. The course of flow showed non-stationary flow at certain travel lengths. It was further proved that the issues of flowing through very narrow slots can be dealt with even though it is difficult. However, a sensitivity analysis or comparison with the experiment has not been performed so far and these would tell more about the accuracy of the solution in these parts of the balanced valve.

Using the obtained results of simulation a force characteristic of the valve was drawn up. It shows the force load of the shaft which continuously regulates valve travel and is connected to a servo-drive. It was also ascertained that accurate determination of counter pressure at the outlet from the diffuser has a significant effect on determination of the force characteristic. For example, in the case of a high travel the pressure difference of $1 \%$ is characterised by almost $10 \%$ difference in the force on the shaft from the aerodynamic forces. The used mesh may also significantly affect the results of the simulation. In the case of these complex models it is necessary to have suitable software for quick generation of suitable and quality computational mesh.

The force characteristic obtained by numerical simulations has been compared with the characteristic obtained by means of experimental measuring. It was determined that the course of the force ascertained from simulation is similar to the course of the force ascertained in the experiment. Similar means a similar tendency which is globally descending. Some areas are also locally similar - those which characterize an unbalanced area, where the force to the shaft suddenly grows. The area of forces where these characteristics are present are also very similar. Absolute values of forces will also have to be further compared with the results of the continuing improved measuring and further with the results on a different computational mesh.

Another topic for further continuing of the research supported by numerical modelling of flow through a balanced valve is also the performance of a greater number of simulations for the area between $10-16 \mathrm{~mm}$, where individual chambers are opened and closed. Further then the performance of sensitivity analysis of effect of slot clearance on the course of flow and also determination of effect of the computing mesh on the course of the flow for this type of balanced valve.

\section{References}

1. R. Matas, L. Bednář, L. Tajč, Experimental Fluid Mechanics 2010 Conference Proceedings, 398-409 (2010)

2. M. Hajšman, D. Kovandová, R. Matas, Experimental Fluid Mechanics 2011 Conference Proceedings, 2, 642-653 (2011)

3. H.Y. Yong, Y.S. Kune, Nuclear Engineering and Design 241, 4061- 4078 (2011)

\section{Acknowledgement}

These results were achieved in the framework of the 1M06059 project of the programme "Research Centres", Ministry of Education of the Czech Republic programme and in specific research (project ZCU SGS-2012-072).

The access to the MetaCentrum computing facilities provided under the programme "Projects of Large Infrastructure for Research, Development, and Innovations" LM2010005 funded by the Ministry of Education, Youth, and Sports of the Czech Republic is acknowledged. 